\title{
Pulsar binary systems in a nonsymmetric theory of gravitation II. Dipole radiation
}

\author{
S. Ragusa* \\ Instituto de Física de São Carlos, \\ Universidade de São Paulo \\ CEP 369, 13560-970 São Carlos, SP, Brazil
}

(Received on 15 January, 2010)

\begin{abstract}
This paper deals with the emission of gravitational radiation in the context of a previously studied metric nonsymmetric theory of gravitation. The part coming from the symmetric part of the metric coincides with the mass quadrupole moment result of general relativity. The one associated to the antisymmetric part of the metric involves the dipole moment of the fermionic charge of the system. The results are applied to binary star systems and the decrease of the period of the elliptical motion is calculated.
\end{abstract}

Keywords: dipole radiation, nonsymmetric

\section{INTRODUCTION}

In previous papers [1] we have studied a metric nonsymmetric theory of gravitation. In the flat space linear approximation [1-I] the antisymmetric part of the metric satisfies Maxwell's type vacuum equations, describing then a spin-1 field. Furthermore, the theory was shown to be free of ghost negative-energy modes when expanded about a Riemannian curved space, being outside of the ill-behaved nonsymmetric theories analyzed by Damour, Deser and McCarthy [2].

After establishing the field equations in [1-I] their solution for a point source mass was obtained in [1-II], together with its consequences for the motion of test particles and light. The theory was shown to be consistent with the four classical solar tests of general relativity (GR). Next in [3] the conservation laws associated to the theory were studied. In [4] we have proved the analogue of the GR static theorem, that is, that the field outside of a time-dependent spherically symmetric source is necessarily static. More recently [5] a post-Newtonian approximation of the theory was developed with the purpose of application to pulsar binary star systems.

The sources of the field are the energy-momentum-stress tensor $T^{\alpha \beta}$ and the fermionic current density $S^{\alpha}$ found, for instance, in the description of the interior of stars (electrons, protons and neutrons).

In this work we investigate the emission of gravitational radiation predicted by the theory. It is shown that the emitted radiation consists of two parts, one coming from the mass quadrupole of the source and the other from its fermion dipole moment. The first one comes from the symmetric part of the metric, which coincides with the GR result, and the other from the antisymmetric part.

The results are considered for a binary pulsar system. As it is known in GR [6] the internal motion of the system are ellipses described by its components and the loss of energy by emission of radiation produces a decrease of the orbital period of the elliptical motion of the system. This is an astronomical observed effect and we want to analyze. With the results obtained in [5] we calculate the contribution of the dipole radiation to the GR result for the secular decrease of the orbital period. This contribution contains the fermionic

${ }^{*}$ Electronic address: ragusa@if.sc.usp.br charges of the pulsar and of its companion. Information about these quantities for particular binaries can also be obtained by analyzing the contribution to the GR values for the precession of the periastron and for the Doppler-red-shift parameter. What their contributions will be is a topic for future investigation.

In Sec. II we present the field equations. In Sec. III we use the energy- momentum-stress pseudotensor derived in [3] to calculate the rate of emitted radiation. After elaborating the weak-field expansion in Sec. IV the rate of radiation, the luminosity, is obtained. In Sec. V the results are applied to a binary system and the secular decrease of the orbital period of the pulsar is calculated. In Sec. VI we present a summary of our conclusions and highlight future work.

\section{FIELD EQUATIONS}

The field equations of the theory are [1-1]

$$
\begin{gathered}
U_{\alpha \beta}+\Lambda g_{(\alpha \beta)}=\kappa\left(T_{(\alpha \beta)}-\frac{1}{2} g_{(\alpha \beta)} T\right), \\
\Gamma_{[\alpha, \beta]}+\Lambda g_{[\alpha \beta]}=\kappa\left(T_{[\alpha \beta]}-\frac{1}{2} g_{[\alpha \beta]} T\right) \\
\mathbf{g}^{(\alpha \beta)}{ }_{\gamma}+\mathbf{g}^{(\alpha \sigma)} \Gamma_{(\sigma \gamma)}^{\beta}+\mathbf{g}^{(\beta \sigma)} \Gamma_{(\sigma \gamma)}^{\alpha}-\mathbf{g}^{(\alpha \beta)} \Gamma_{(\sigma \gamma)}^{\sigma}=0,
\end{gathered}
$$

and

$$
\mathbf{g}^{[\alpha \beta]}{ }_{, \beta}=4 \pi G \mathbf{S}^{\alpha} .
$$

The notation () and [] designates symmetric and antisymmetric parts. In the first equation $\kappa=8 \pi G$ as usual and

$$
U_{\alpha \beta}=\Gamma_{(\alpha \beta), \sigma}^{\sigma}-\Gamma_{(\beta \sigma), \alpha}^{\sigma}+\Gamma_{(\alpha \beta)}^{\sigma} \Gamma_{(\rho \sigma)}^{\rho}-\Gamma_{(\alpha \rho)}^{\sigma} \Gamma_{(\sigma \beta)}^{\rho},
$$

symmetric because the second term is (see just after (2.10)). $\Lambda$ is the cosmological constant and $T=g^{\rho \sigma} T_{\rho \sigma}$. In equation (2.2), $\Gamma_{[\alpha, \beta]}$ is the curl of the vector $\Gamma_{\alpha}=\Gamma_{[\alpha \mu]}^{\mu}$ which acts then as a vector potential. As it is defined up to a gradient we can choose the gauge

$$
\partial^{\alpha} \Gamma_{\alpha}=0 .
$$


In (2.3) and (2.4) we use the notation $\mathbf{X}=\sqrt{-g} X$ with $g=\operatorname{det}\left(g_{\alpha \beta}\right)$ and $g^{\alpha \beta}$ is the inverse of $g_{\alpha \beta}$ as defined by

$$
g^{\alpha \beta} g_{\alpha \gamma}=g^{\beta \alpha} g_{\gamma \alpha}=\delta_{\gamma}^{\beta} .
$$

¿From (2.4) we have

$$
\partial_{\alpha} \mathbf{S}^{\alpha}=0
$$

the equation of continuity for the fermionic current, saying that

$$
F=G \int \mathbf{S}^{0} d^{3} x
$$

is a constant. This is the fermion charge of the system. From (2.4) its dimension is of square-length.

Equation (2.3) can be solved for the symmetric part of the connection (1-I) giving,

$$
\begin{gathered}
\Gamma_{(\alpha \beta)}^{\sigma}=\frac{1}{2} g^{(\sigma \gamma)}\left(s_{\alpha \gamma, \beta}+s_{\beta \gamma, \alpha}-s_{\alpha \beta, \gamma}\right) \\
+\frac{1}{4}\left(g^{(\sigma \gamma)} s_{\alpha \beta}-\delta_{\alpha}^{\sigma} \delta_{\beta}^{\gamma}-\delta_{\alpha}^{\gamma} \delta_{\beta}^{\sigma}\right)\left(\ln \frac{s}{g}\right)_{, \gamma},
\end{gathered}
$$

where $s_{\alpha \beta}$, symmetric and with determinant $s$, is the inverse of $g^{(\alpha \beta)}$ as defined by $s_{\alpha \beta} g^{(\alpha \gamma)}=\delta_{\beta}^{\gamma}$. In deriving Eq. (2.10) we come across the relation $\Gamma_{(\beta \sigma)}^{\sigma}=\partial_{\beta} \ln (-g / \sqrt{-s})$, which can be re-obtained from that equation. We then see that the second term on the right of (2.5) is in fact symmetric.

\section{THE LUMINOSITY}

The total energy-momentum-stress divergence equation in the theory is [3]

$$
\left(\mathbf{T}_{\alpha}{ }^{\sigma}+\mathbf{t}_{\alpha}{ }^{\sigma}\right), \sigma=0
$$

where

$$
\mathbf{T}_{\alpha}{ }^{\sigma} \equiv \frac{1}{2}\left(g_{\alpha \nu} \mathbf{T}^{\sigma v}+g_{v \alpha} \mathbf{T}^{v \sigma}\right)
$$

and

$$
\begin{aligned}
\mathbf{k t}_{\alpha}{ }^{\sigma}= & \frac{1}{2}\left(\Gamma_{(\beta \gamma)}^{\sigma} \mathbf{g}^{(\beta \gamma)}{ }_{\alpha}-\Gamma_{(\gamma \rho)}^{\rho} \mathbf{g}^{(\sigma \gamma)}, \alpha\right)-\Gamma_{[\alpha, \beta]} \mathbf{g}^{[\sigma \beta]} \\
& +\frac{1}{2} \delta_{\alpha}^{\sigma}\left(\mathbf{L}^{\prime}+\Gamma_{[\beta, \gamma]} \mathbf{g}^{[\beta \gamma]}+2 \Lambda \sqrt{-g}\right),
\end{aligned}
$$

where

$$
\mathbf{L}^{\prime}=\mathbf{g}^{(\alpha \beta)}\left(\Gamma_{(\alpha \rho)}^{\sigma} \Gamma_{(\sigma \beta)}^{\rho}-\Gamma_{(\alpha \beta)}^{\sigma} \Gamma_{(\sigma \rho)}^{\rho}\right)
$$

$T^{\mu \nu}$ is the upper indices matter stress tensor, with which the down indices $T_{\alpha \beta}$ is related to by

$$
T_{\alpha \beta}=g_{\alpha v} g_{\mu \beta} T^{\mu \nu}
$$

and $\mathbf{t}_{\alpha}{ }^{\sigma}$ is the generalized gravitational stress pseudotensor.
Integration of (3.1) for $\alpha=0$ in a volume containing the localized source gives

$$
-\frac{d}{d t} \int_{V}\left(\mathbf{T}_{0}{ }^{0}+\mathbf{t}_{0}{ }^{0}\right) d V=\int_{S}\left(\mathbf{T}_{0}{ }^{i}+\mathbf{t}_{0}{ }^{i}\right) n^{i} d A,
$$

where $S$ is the surface that involves the volume $V$ and $n^{i}$ is the normal to the surface with area element $d A$. The first term is the rate of decrease of the total energy $P^{0}$,

$$
P^{0}=\int_{V}\left(\mathbf{T}_{0}^{0}+\mathbf{t}_{0}{ }^{0}\right) d V .
$$

Taking $S$ outside the localized matter source, in the radiation zone, equation (3.6) says that the rate of decrease is given by the flux of $\mathbf{t}_{0}{ }^{i}$, which is the generalized gravitational Poynting vector. Then the rate at which the system looses energy due to gravitational waves generated by the internal motion of its components is given by

$$
-\frac{d P^{0}}{d t}=\frac{d E}{d t}
$$

where, with the sources located around the origin, the gravitational waves energy rate is given by

$$
\frac{d E}{d t}=\int_{(\infty)} \mathbf{t}_{0}{ }^{i} n^{i} r^{2} d \Omega .
$$

Here $r=\mid \mathbf{r}$ । is the radius of $S$ at infinity and $n^{i}=x^{i} / r$ is its normal. This is the generalized luminosity formula. From (13)

$$
\mathbf{t}_{0}{ }^{i}=\frac{1}{16 \pi G}\left(\Gamma_{(\beta \gamma)}^{i} \mathbf{g}^{(\beta \gamma)}{ }_{0}-\Gamma_{(\gamma \rho)}^{\rho} \mathbf{g}^{(i \gamma)}, 0-2 \Gamma_{[0, j]} \mathbf{g}^{[i j]}\right) .
$$

From (3.9) we see that this quantity needs to be calculated only to order $r^{-2}$ at infinity. Therefore, as it is quadratic in the fields we will need the fields only to order $r^{-1}$. Also, only to order $G$ to have the luminosity to that order.

As for $P^{0}$ the integrand in (17) contains the two terms

$$
\left.\mathbf{T}_{0}^{0}=\sqrt{-g}\left(g_{(0 v)} T^{(0 v)}+g_{[0 v]} T^{[0 v]}\right)\right)
$$

and

$$
\mathbf{t}_{0}{ }^{0}=\frac{1}{16 \pi G}\left(\Gamma_{(\beta \gamma)}^{0} \mathbf{g}^{(\beta \gamma)}{ }_{0}+\Gamma_{(\gamma \rho)}^{\rho} \mathbf{g}^{(0 \gamma)}, 0+\mathbf{L}^{\prime}+\Gamma_{[i, j]} \mathbf{g}^{[i j]}\right),
$$

where we have neglected here the $\Lambda$ term as in GR. With the fields calculated to order $G$ we will have also the total energy $P^{0}$ to that order.

For a star binary system that we are interested in, the internal motion are ellipses described by its components and the loss of energy $P^{0}$ will produce a decrease of the orbital period $P$ of the elliptical motion of the system. To obtain it we go to the weak-field expansion in the next section.

\section{WEAK-FIELD EXPANSION}

We now expand the field equations about a flat space-time background by putting

$$
g_{\alpha \beta}=\eta_{\alpha \beta}+h_{\alpha \beta},
$$


where $\eta_{\alpha \beta}$ is the Minkowski metric $\operatorname{diag}(+1,-1,-1,-1)$ and $\left|h_{\alpha \beta}\right|<<1$. Then to linear order we have, dispensing with $O\left(h^{2}\right)$,

$$
\sqrt{-g}=1+\frac{1}{2} h
$$

where $h=\eta^{\alpha \beta} h_{(\alpha \beta)}$. From (7)

$$
g^{\alpha \beta}=\eta^{\alpha \beta}-h^{\beta \alpha},
$$

where indices are raised and lowered with $\eta$ i.e., $h_{\lambda}^{\alpha}=$ $\eta^{\alpha \beta} h_{\lambda \beta}$. Thence,

$$
g^{(\alpha \beta)}=\eta^{\alpha \beta}-h^{(\alpha \beta)} ; \quad g^{[\alpha \beta]}=h^{[\alpha \beta]} .
$$

For the inverse of $g^{(\alpha \beta)}$ we have

$$
s_{\alpha \beta}=\eta_{\alpha \beta}+h_{(\alpha \beta)} \text {. }
$$

From here the linear part of (10) is

$$
\Gamma_{(\alpha \beta)}^{\sigma}=\frac{1}{2} \eta^{\rho \sigma}\left(h_{(\alpha \rho), \beta}+h_{(\beta \rho), \alpha}-h_{(\alpha \beta), \rho}\right) .
$$

because $\ln (s / g)$ is of second order in the fields. In fact, we have the relations $g^{-1}=\varepsilon_{\alpha \beta \gamma \delta} \varepsilon_{\mu v \rho \sigma} g^{\alpha \mu} g^{\beta v} g^{\gamma \rho} g^{\delta \sigma} / 4$ ! and $s^{-1}=\varepsilon_{\alpha \beta \gamma \delta} \varepsilon_{\mu v \rho \sigma} g^{(\alpha \mu)} g^{(\beta v)} g^{(\gamma \rho)} g^{(\delta \sigma)} / 4$ !. Writing $g^{\alpha \beta}=$ $g^{(\alpha \beta)}+g^{[\alpha \beta]}$ we find, to lowest order, $g^{-1}=s^{-1}-h^{[\mu v]} h_{[\mu v]} / 2$ or $s / g=1+h^{[\mu v]} h_{[\mu v]} / 2$. Therefore, $\ln (s / g)=h^{[\mu v]} h_{[\mu v]} / 2$ and (4.6) follows. Then,

$$
\Gamma_{(\alpha \sigma)}^{\sigma}=\frac{1}{2} h, \alpha
$$

Separating the symmetric and antisymmetric contributions in (10) we have to the considered order

$$
\mathbf{t}_{0}^{i}=\mathbf{t}_{0}{ }^{i}(1)+\mathbf{t}_{0}^{i}(2),
$$

where

$$
\begin{aligned}
& 16 \pi G \mathbf{t}_{0}{ }^{i}(1)=\frac{1}{2} h_{(\beta \gamma),}{ }^{i} h_{0}^{(\beta \gamma)}-h_{\beta), \gamma}^{(i} h_{, 0}^{(\beta \gamma)} \\
& +\frac{1}{2}\left(\frac{1}{2} h^{i} h_{, 0}-h_{, \gamma} h_{, 0}^{(i \gamma)}\right)
\end{aligned}
$$

and

$$
8 \pi G \mathbf{t}_{0}{ }^{i}(2)=-\Gamma_{[0, j]} h^{[i j]} .
$$

The luminosity in (3.9) splits in the two corresponding terms

$$
\frac{d E}{d t}=\frac{d E_{1}}{d t}+\frac{d E_{2}}{d t} .
$$

The first contribution is

$$
\frac{d E_{1}}{d t}=\int_{(\infty)} \mathbf{t}_{0}^{i}(1) n^{i} r^{2} d \Omega .
$$

In the Appendix we show that this contribution reproduces the GR mass quadrupole result.

$$
\frac{d E_{1}}{d t}=\frac{G}{5} \frac{d^{3} Q_{i j}}{d t^{3}} \frac{d^{3} Q_{i j}}{d t^{3}},
$$

where

$$
Q_{i j}(t)=\int \rho(\mathbf{r}, t)\left(x_{i} x_{j}-\frac{1}{3} r^{2} \delta_{i j}\right) d V
$$

is the traceless mass quadrupole moment to be taken at the retarded time $t-r$.

Let us consider now the second contribution

$$
\frac{d E_{2}}{d t}=\int_{(\infty)} \mathbf{t}_{0}{ }^{i}(2) n^{i} r^{2} d \Omega .
$$

As we are outside the sources and at infinity equation (2) tell us that equation (32) can be written

$$
\kappa \mathbf{t}_{0}{ }^{i}(2)=\Lambda h_{[0 j]} h^{[i j]} .
$$

For a system of particles $T^{[\mu v]}$ is null. Thence, the righthand-side of (2) to first order in $G$ is, from (15), equal to $\kappa T_{[0 i]}^{(0)}=\kappa \eta_{0 v} \eta_{\mu i} T^{[\mu v](0)}=0$. Therefore, the equation reduces to

$$
\Gamma_{[\alpha, \beta]}+\Lambda h_{[\alpha \beta]}=0
$$

in all space. Equation (2.4) to first order is

$$
h^{[\alpha \beta]}{ }_{, \beta}=4 \pi G S^{\alpha} .
$$

Taking the divergence of (39) and using the second we obtain, from (6),

$$
\frac{1}{2} \partial^{\beta} \partial_{\beta} \Gamma_{\alpha}+4 \pi G \Lambda S_{\alpha}=0
$$

The solution is

$$
\Gamma_{\alpha}(\mathbf{r}, t)=-2 \Lambda G \int \frac{S_{\alpha}\left(\mathbf{r}^{\prime}, t-\left|\mathbf{r}-\mathbf{r}^{\prime}\right|\right.}{\left|\mathbf{r}-\mathbf{r}^{\prime}\right|} d^{3} x^{\prime}
$$

Far away we have, with $t^{*}=t-r$ being the retarded time with respect to the origin around which the source is located, we have to dipole order

$$
\Gamma_{\alpha}(\mathbf{r}, t)=-\frac{2 \Lambda G}{r} \int\left(S_{\alpha}\left(\mathbf{r}^{\prime}, t^{*}\right)+n^{i} \dot{S}_{\alpha}\left(\mathbf{r}, t^{*}\right) x^{i}\right) d^{3} x^{\prime},
$$

where $\dot{S}_{\alpha}=d S_{\alpha} / d t^{*}$. Therefore the time component is, with (9),

$$
\Gamma_{0}(\mathbf{r}, t)=-\frac{2 \Lambda}{r}\left(F-n^{i} \dot{p}^{i}\left(t^{*}\right)\right),
$$

where $F$ is the fermion charge and

$$
p^{i}(t)=G \int x^{i} S^{0}(\mathbf{r}, t) d^{3} x,
$$

is the fermionic dipole moment. For the space component we have

$$
\Gamma_{i}(\mathbf{r}, t)=\frac{2 \Lambda G}{r} \int S^{i}\left(\mathbf{r}^{\prime}, t^{*}\right) d^{3} x^{\prime}
$$


From (2.9), $x^{i} S^{\mu},{ }_{\mu}=0$ or $x^{i} S^{0}{ }_{0}+\left(x^{i} S^{j}\right),{ }_{j}-S^{i}=0$. Thence

$$
\Gamma_{i}(\mathbf{r}, t)=\frac{2 \Lambda}{r} \dot{p}^{i}
$$

Taking (44) and (46) in the time-space component of (39) we have, noting that $\partial_{i} \dot{p}^{j}\left(t^{*}\right)=\ddot{\mathbf{p}}^{j} n^{i}$,

$$
h_{[0 j]}=\frac{1}{r}\left(\ddot{p}^{j}-\mathbf{n} \cdot \ddot{\mathbf{p}}_{n^{j}}\right) .
$$

For the space-space component

$$
h_{[i j]}=\frac{1}{r}\left(\ddot{p}^{i} n^{j}-\ddot{p}^{j} n^{i}\right) .
$$

With these expressions (38) gives, after contraction with $n^{i} r^{2}$

$$
\kappa \mathbf{t}_{0}{ }^{i}(2) n^{i} r^{2}=-\Lambda\left(\ddot{\mathbf{p}}^{2}-n^{i} n^{j} \ddot{p}^{j} \ddot{p}^{i}\right)
$$

As the angular integral of $n^{i} n^{j}$ is equal to $4 \pi \delta_{i j} / 3$ we find

$$
\frac{d E_{2}}{d t}=\frac{1}{3 G}|\Lambda| \ddot{\mathbf{p}}^{2},
$$

where we have taken $\Lambda<0$ in agreement with [1-I]

\section{THE BINARY PULSAR SYSTEM}

We consider now a binary system and make use of the total energy of the system obtained in [5],

$$
P^{0}=m_{1}+m_{2}+\frac{1}{2} m_{1} v_{1}^{2}+\frac{1}{2} m_{2} v_{2}^{2}-G \frac{m_{1} m_{2}}{r},
$$

where $r=\mid \mathbf{x}_{1}(t)-\mathbf{x}_{2}(t)$ । is the distance between the particles.

For the bound elliptical motion of the system we have

$$
P^{0}=m_{1}+m_{2}+\frac{1}{2}\left(m_{1}+m_{2}\right) V^{2}+E_{I}
$$

where $V$ is the constant velocity of the center of mass of the system and

$$
E_{I}=-\frac{G m_{1} m_{2}}{2 a}
$$

is its internal energy, $a$ being the semimajor axis of the relative elliptical motion. From Kepler's third law,

$$
\left(\frac{P}{2 \pi}\right)^{2}=\frac{a^{3}}{G\left(m_{1}+m_{2}\right)} .
$$

As argued in GR, due to the emission of radiation the ellipsis will be deformed with time variation of $a$ and $P$. Differentiation of the above relations and eliminating the term $d a / d t$ it follows, with $d E_{I} / d t=d P^{0} / d t$ from (53),

$$
\frac{1}{P} \frac{d P}{d t}=\frac{3 a}{G m_{1} m_{2}} \frac{d P^{0}}{d t} .
$$

Therefore, with (3.8) the decrease of the period $P$ due to the emission of radiation is given by,

$$
\frac{1}{P} \frac{d P}{d t}=-\frac{3 a}{G m_{1} m_{2}}\left(\frac{d E_{1}}{d t}+\frac{d E_{2}}{d t}\right) .
$$

Let $\mathbf{r}_{1}$ and $\mathbf{r}_{2}$ be the positions of the pulsar and of its companion measured from the center of mass of the system, and $\mathbf{r}_{=} \mathbf{r}_{1-} \mathbf{r}_{2}$. We then have $m_{1} \mathbf{r}_{1}+m_{2} \mathbf{r}_{2}=0$ and in terms of the total mass $M=m_{1}+m_{2}$,

$$
\mathbf{r}_{1}=\frac{m_{2}}{M} \mathbf{r} ; \quad \mathbf{r}_{2}=-\frac{m_{1}}{M} \mathbf{r} .
$$

¿From here the fermionic dipole moment of the system is, $\mathbf{p}=F_{1} \mathbf{r}_{1}+F_{2} \mathbf{r}_{2}$ or

$$
\mathbf{p}=\mu D \mathbf{r}
$$

where $\mu=m_{1} m_{2} / M$ is the reduced mass and

$$
D=\frac{F_{1}}{m_{1}}-\frac{F_{2}}{m_{2}}
$$

is the dipole parameter.

The Keplerian orbit for the system, with eccentricity $e$ is given by

$$
r=\frac{a\left(1-e^{2}\right)}{1+e \cos \varphi}
$$

$$
\dot{\varphi}=\frac{d \varphi}{d t}=\frac{\left(G M a\left(1-e^{2}\right)\right)^{1 / 2}}{r^{2}},
$$

where $\varphi$ is the angle between $\mathbf{r}$ and the periastron direction. The rate of energy lost by the system is given by the sum of the results in equations (35) and (51). The first one gives the GR result, the average over one period being [8],

$$
\left\langle\frac{d E_{1}}{d t}\right\rangle=\frac{32}{5} \frac{G^{4} m_{1}^{2} m_{2}^{2}\left(m_{1}+m_{2}\right)}{a^{5}} f_{G R}(e),
$$

where

$$
f_{G R}(e)=\left(1-e^{2}\right)^{-7 / 2}\left(1+\frac{73}{24} e^{2}+\frac{37}{96} e^{4}\right) .
$$

For the second one the average is

$$
\left\langle\frac{d E_{2}}{d t}\right\rangle=\frac{1}{P} \int_{0}^{2 \pi} \frac{d E_{2}}{d t} \frac{d t}{d \varphi} d \varphi .
$$

Using (62) we obtain

$$
\left\langle\frac{d E_{2}}{d t}\right\rangle=\frac{|\Lambda|}{3 a^{4}} G m_{1}^{2} m_{2}^{2} D^{2} f_{d}
$$

where

$$
f_{d}=\left(1-e^{2}\right)^{-5 / 2}\left(1+\frac{e^{2}}{2}\right) .
$$

With these results the secular decrease of the orbital period $P$ is give by

$$
\frac{\dot{P}}{P}=\left(\frac{\dot{P}}{P}\right)_{G R}+\left(\frac{\dot{P}}{P}\right)_{d},
$$

where

$$
\left(\frac{\dot{P}}{P}\right)_{G R}=-\frac{96}{5}\left(\frac{2 \pi}{P}\right)^{8 / 3} \frac{G^{4} m_{1} m_{2}}{\left(m_{1}+m_{2}\right)^{1 / 3}} f_{G R}(e)
$$


and

$$
\left(\frac{\dot{P}}{P}\right)_{d}=-\left(\frac{2 \pi}{P}\right)^{2}|\Lambda| \frac{m_{1} m_{2}}{G\left(m_{1}+m_{2}\right)}\left(\frac{F_{1}}{m_{1}}-\frac{F_{2}}{m_{2}}\right)^{2} f_{d}(e) .
$$

Due to the presence of the cosmological constant the contribution of the dipole is not expected to be important for a determination of the fermion charges of a given system. For this purpose we will then analyze in a third paper the decrease of the precession of the periastron of the binary system and of the Doppler-red-shift parameter.

\section{CONCLUSION}

Giving sequence to the study of a metric nonsymmetric theory of gravitation we have discussed the emission of gravitational radiation. The out put power of emission contains two terms, one involving the symmetric part of the metric and the other its antisymmetric part. The first one agrees with the mass quadrupole result of GR and the other is a dipole emission due to the fermionic current of the system.

The results have been applied to a star binary system and the decrease of the orbital period of the system has been calculated. The dipole contribution contains the fermion charges $F_{p}$ and $F_{c}$ of the pulsar and its companion. As the dipole part is proportional to the cosmological constant the result of the calculation indicates that the dipole part is negligible. Additional information is then needed for the determination of the fermion charges of a given system. This can be obtained by analyzing their contribution to the GR values for the decrease of the precession of the periastron of the binary system, and of the Doppler-red-shift parameter. What their contributions will be is a topic for future work.

\section{APPENDIX. DERIVATION OF (35)}

Equation (1) gives to lowest order,and neglecting the contribution of the cosmological constant as in the corresponding equation in GR,

$$
U_{\alpha \beta}=\kappa\left(T_{(\alpha \beta)}^{0}-\frac{1}{2} \eta_{\alpha \beta} \eta^{\gamma \lambda} \stackrel{0}{T}_{(\gamma \lambda)}\right)
$$

where $\stackrel{0}{T}_{(\alpha \beta)}$ is the matter tensor of order zero, with no interactions, of special relativity. By contracting with $\eta^{\alpha \beta}$ and going back, we obtain

$$
U_{\alpha \beta}-\frac{1}{2} \eta_{\alpha \beta} U=\kappa \stackrel{0}{T}_{(\alpha \beta)}=\kappa \eta_{\alpha \rho} \eta_{\sigma \beta} \stackrel{0}{T}^{(\rho \sigma)},
$$

where in the last step we have gone to the upper indices energy tensor by (13). From here contraction with $\eta^{\alpha v} \eta^{\mu \beta}$ gives

$$
\eta^{\alpha v} \eta^{\mu \beta} U_{\alpha \beta}-\frac{1}{2} \eta^{\mu v} U=\kappa T^{(\mu v)} .
$$

With equations (28) and (29), we have

$$
U_{\alpha \beta}=\frac{1}{2}\left(h_{(\alpha}{ }^{\sigma}{ }_{\beta, \sigma}+h_{(\beta}{ }^{\sigma)}{ }_{, \alpha \sigma}-h_{, \alpha \beta}-h_{(\alpha \beta), \sigma}{ }^{\sigma}\right)
$$

and from here,

Substituting in (73)

$$
U=\eta^{\alpha \beta} U_{\alpha \beta}=h_{, \beta \sigma}^{(\beta \sigma)}-h_{, \sigma}{ }^{\sigma} .
$$

$h^{(\mu \sigma), \nu}{ }^{\nu} \sigma+h^{(v \sigma)},_{\sigma}^{\mu}-h^{\mu \nu}-h^{(\mu \nu)}, \sigma{ }^{\sigma}-\eta^{\mu \nu}\left(h^{(\rho \sigma)}{ }_{, \rho \sigma}-h_{, \sigma}{ }^{\sigma}\right)$

$$
=2 \kappa \stackrel{0}{T}^{(\mu v)} \text {. }
$$

From this we have the free matter conservation law

$$
\partial_{\mu} \stackrel{0}{(\mu v)}^{(\mu)} 0
$$

Calling

$$
\Theta^{\mu v}=h^{(\mu v)}-\frac{1}{2} \eta^{\mu v} h
$$

and adopting the Hilbert gauge

$$
\Theta^{\mu \nu},_{\nu}=0 \text {, }
$$

we have

$$
\partial^{\sigma} \partial_{\sigma} \Theta^{\mu v}=2 \kappa \stackrel{0}{T}^{(\mu v)}
$$

The solution of this equation with an outgoing boundary condition is

$$
\Theta^{\mu v}(\mathbf{r}, t)=-\frac{\kappa}{2 \pi} \int \frac{1}{\left|\mathbf{r}-\mathbf{r}^{\prime}\right|} T^{0}(\mu v)\left(\mathbf{r}^{\prime}, t-\left|\mathbf{r}-\mathbf{r}^{\prime}\right|\right) d^{3} x^{\prime}
$$

At points far from the source $(r>>d=$ linear dimension of source, ) and keeping only the first term of the expansion of the integrand we have

$$
\Theta^{\mu v}(\mathbf{r}, t)=-\frac{\kappa}{2 \pi r} \psi^{\mu v}(t-r),
$$

where

$$
\psi^{\mu v}(t-r)=\int T^{0}(\mu v)(\mathbf{r}, t-r) d V^{\prime}
$$

at the retarded time $t-r$ relative to the origin. We can now calculate the first contribution to the luminosity in (34). Using in (31) the inverse of (78),

$$
h^{(\mu v)}=\Theta^{\mu v}-\frac{1}{2} \eta^{\mu v} \Theta
$$

where $\Theta=\eta_{\alpha \beta} \Theta^{\alpha \beta}$, equation (35) follows.
[1] S. Ragusa, Phys. Rev. D 56, 864 (1997).. The term $2 \Delta_{\alpha} / 3$ of equation (6.7) is here replaced by $\Gamma_{\alpha}$; Gen. Relat. Gravit. 31 275 (1999). These papers will be referred to as I and II, respec- tively.

[2] T. Damour, S. Deser, and J. McCarthy, Phys. Rev. D 45, R3289 (1992); 47, 1541 (1993). 
[3] S. Ragusa, Braz. J. Phys. 35, 1020 (2005).

[4] S. Ragusa and F. Bosquetti, Braz. J. Phys. 36, 1223 (2006).

[5] S. Ragusa, submitted.

[6] See for instance, N Straumann, General Relativity and
Relativistic Astrophysics, Springer-Verlag 1984.

[7] P. C. Peters and J. Mathews, Phys. Rev. 131, 435 (1963). 